\title{
Dissecting aneurysms of the vertebral arteries following cervical manipulation: A case report
}

\author{
JOHN W DUNNE, G NEIL CONACHER,* MAKHAN KHANGURE, \\ CLIVE G HARPER† \\ From the Departments of Neurology, Neuropathology, Radiology, Royal Perth Hospital, Perth, Australia
}

SUMMARY Neck manipulation may uncommonly be associated with serious and even fatal vascular complications. Although well recognised, the nature of the vascular injury has only rarely been directly established by pathological examination. The case is reported of a 43-year-old man who died following neck manipulation, and in whom multiple dissecting aneurysms within both vertebral arteries were demonstrated radiologically and found at necropsy. Bilateral dissecting aneurysms were found both at the level of atlanto-axial articulation and close to the origins of the vertebral arteries. No predisposition was found, other than early atheroma consistent with the patient's age.

Alternative medicine, including chiropractic and osteopathy, enjoys an ever-increasing popularity. The Australian Health Survey for 1977-1978 estimated that in a four-week period just over $2 \%$ of Australians had consulted a chiropractor, naturopath or osteopath. $^{1}$ However, an increasing number of reports indicate that spinal manipulation can be associated with serious and even fatal vascular ${ }^{2-14}$ and non-vascular injuries. Vascular injury related to neck manipulation has mainly involved the vertebral and basilar arteries $2-101314$ although several reports describe injuries to the internal carotid artery. ${ }^{11} 12$ Thrombosis and occlusion of the vertebral and basilar- arteries, ${ }^{2-4}$ traumatic vertebral artery pseudo-aneurysm, ${ }^{45}$ haemorrhage from perforation of the vertebral artery, ${ }^{4}$ and vertebral artery dissecting aneurysm ${ }^{6}$ have all been reported, these

\footnotetext{
Address for reprint requests: Dr JW Dunne, Mayo Clinic, 200 1st Street S.W.. Rochester. MN 55905, USA.

*Present address: Kingston Penitentiary Treatment Center, Kingston. Ontario. Canada.

+Present address: Department of Pathology, University of Sydney, Sydney NSW 2006. Australia.
}

Received 20 December 1985 and in revised form 4 June 1986. Accepted 19 June 1986 mainly having been adduced from radiographic findings. The nature of the vascular injury has only rarely been directly established by pathological examination, ${ }^{23414}$ thus prompting this report.

We describe the case of a 43-year-old man who died following neck manipulation and in whom multiple dissecting aneurysms within both vertebral arteries were demonstrated radiographically and found at necropsy.

\section{Case report}

A 43-year-old truck driver presented with a three-month history of intermittent headache and neck discomfort associated with long-distance driving. He went to a naturopath, since he had had similar symptoms treated successfully by local manipulation 3 years before. Immediately after a therapeutic twisting of the neck he experienced the sudden onset of vertigo, with transient loss of consciousness followed by persistent vertigo, vomiting, and oscillopsia. There was no previous history of similar episodes, hypertension, or other significant illness.

Upon examination 6 hours after the onset he was drowsy, but orientated and co-operative. There was no neck stiffness or photophobia. A slurring dysarthria, rotatory nystagmus in the primary position, and mild bilateral upper limb intention tremor were present. The patient was able to sit but was unwilling to stand because of vertigo. There were no cranial or neck bruits. Pulse was $70 /$ minute and regular, blood pressure $130 / 70 \mathrm{mmHg}$, and heart sounds were normal. Radiographs of the cervical spine showed minimal 

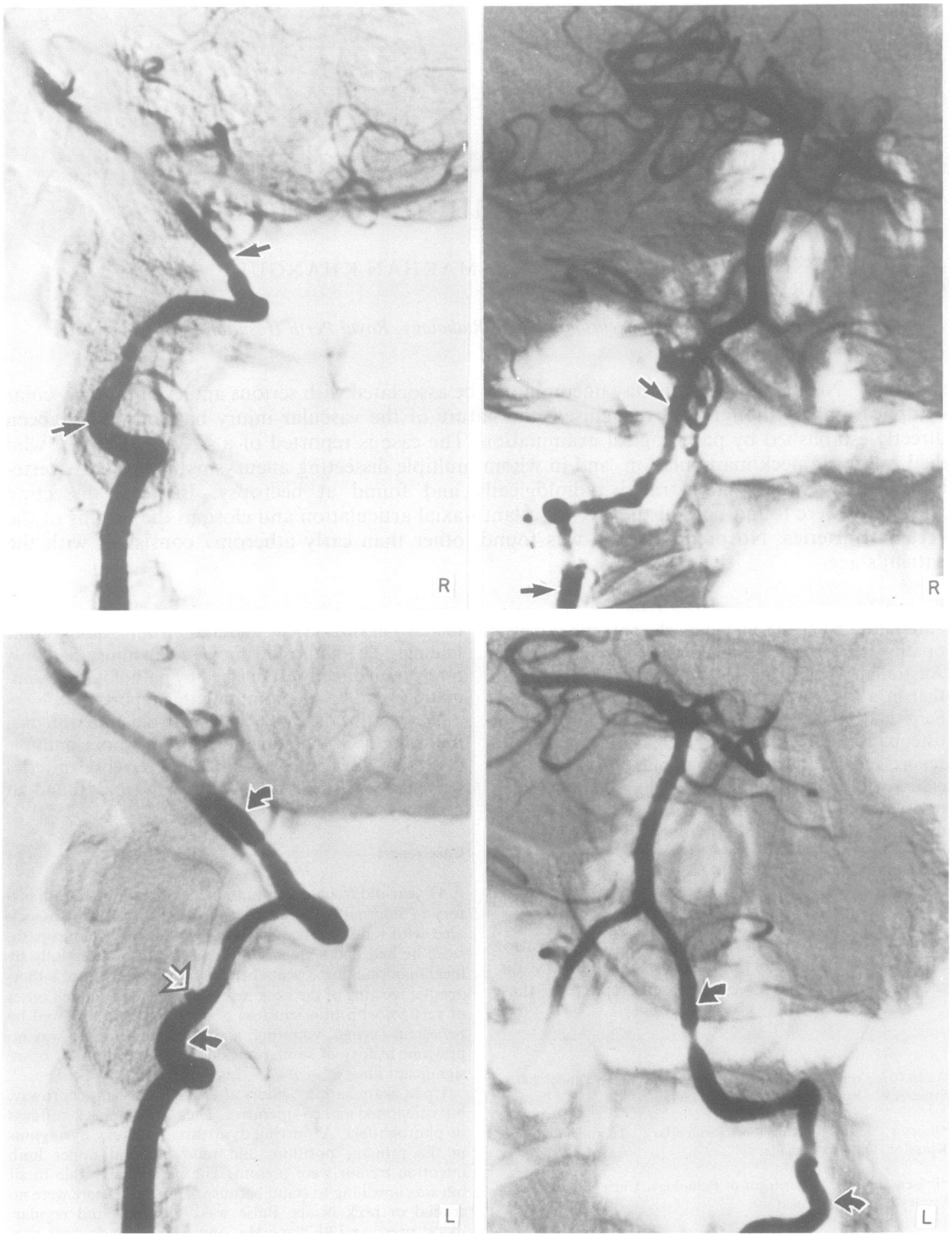

Fig 1 (a) Subtraction print, lateral and AP, of right vertebral artery. The traumatised segment (between arrows) shows narrowing of the lumen and irregularity of the vessel wall, suggesting a dissection. (b) Subtraction print, lateral and AP, of left vertebral artery. In addition to the narrowed vessel lumen (curved arrows) there is a pseudo-aneurysm (open arrow). 
osteophytic lipping of the lower cervical vertebrae but no other abnormalities. Cranial CT scan with and without contrast was normal. Plasma urea and electrolytes, blood sugar level and full blood picture were normal. Syphilis and hepatitis-B serology were negative as was serum anti-nuclear antibody.

Over the next 24 hours the patient remained drowsy with persistent generalised headache. He developed moderate neck stiffness, conjugate deviation of the eyes to the left, a right horizontal gaze palsy and impairment of upgaze. Horizontal jerk nystagmus was noted on left lateral gaze. Tone and deep tendon reflexes of the right arm and leg were augmented, although there was no obvious weakness and plantar responses remained flexor.

Occlusion or dissection of the vertebral arteries was suspected and angiography was performed. Both common carotid and vertebral arteries were catheterised via a femoral approach. The carotid studies were normal. The vertebral arteries both showed abnormalities extending from mid C2 to the level of the foramen magnum. On the right side (fig la) the involved segment (between arrows) showed narrowing of the opacified lumen and irregularity of the vessel wall. On the left (fig lb) there were similar changes, but in addition there was a pseudo-aneurysm (open arrow). The posterior inferior cerebellar arteries (PICAs) were normal as was the basilar artery. These appearances were consistent with bilateral dissections.

The patient was treated conservatively. Forty-two hours after neck manipulation he had a respiratory arrest and required intubation and ventilation. He developed a flaccid quadriplegia and hypotension. Initially brainstem reflexes were present, however these were lost over the next 4 hours. Neurological examination a further day later confirmed that the patient showed the criteria for brainstem death and advanced life support was discontinued.

\section{Pathological findings}

The necropsy was performed by Dr J Hilton, forensic pathologist. General findings were of extensive hypostatic pneumonic consolidation, congestion and oedema. The aorta showed minimal atheroma laid down in streaks, but other major arteries appeared healthy. The brain and spine were referred to the Department of Neuropathology, Royal Perth Hospital, for detailed studies.

Macroscopic examination of the brain showed generalised swelling with flattening of the gyri. The pons, superior medulla, and posterior portion of the left cerebellar hemisphere were markedly softened, and there was bilateral tonsillar herniation through the foramen magnum. Extensive necrosis involving both brainstem and cerebellum was confirmed on microscopic examination. Ischaemic neuronal change was evident in the carotid arterial territories, but without frank necrosis.

The vasculature at the base of the brain was normal with no macroscopic evidence of atheroma. The carotid arteries were also normal throughout their lengths. The vertebral arteries were dissected out from their origins at the subclavian arteries by removal of the anterior arches of the for-

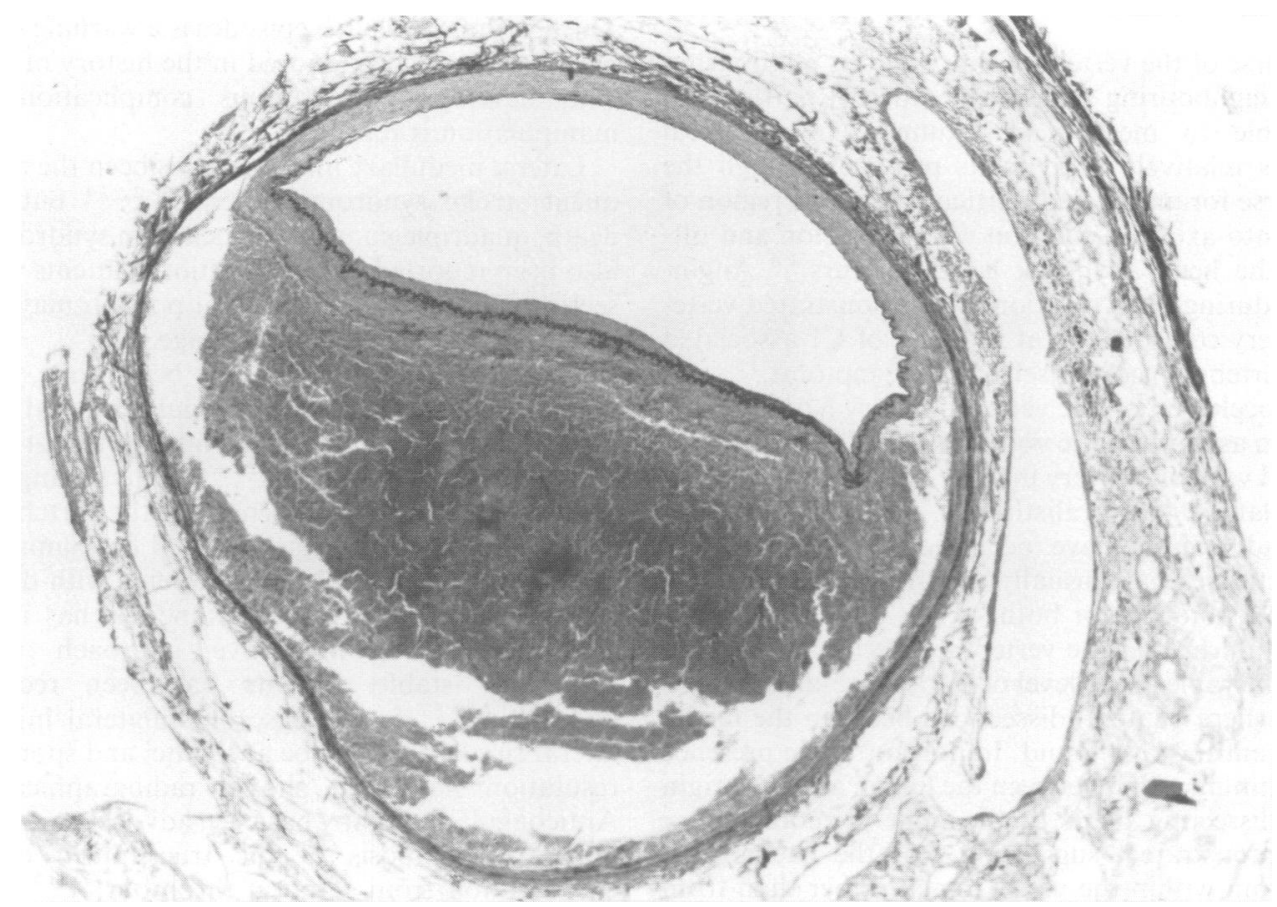

Fig 2 Section of the right vertebral artery at the atlanto-axial articulation, stained to show elastic tissue. Thrombus is seer within the dissection which lies between the tunica media and adventitia. Elastica van Gieson, $\times 10$. 
amina transversaria. The right vertebral artery showed areas of contusion of the adventitia at $7 \mathrm{~cm}$ and $10-12 \mathrm{~cm}$ from its origin. The arteries were sectioned transversely at $1 \mathrm{~cm}$ intervals. Macroscopically, dissecting aneurysms were seen in the right vertebral artery at $0-5 \mathrm{~cm}$ and $9-12 \mathrm{~cm}$ from its origin, and in the left vertebral artery at $1-3 \mathrm{~cm}$ and $11-12 \mathrm{~cm}$. The arterial walls were intact in areas between. Thrombus was present within the dissections with occlusion of the original lumina at the level of the atlanto-axial articulation, $11-12 \mathrm{~cm}$ from the origins of the arteries (fig. 2). Contrast medium from post-mortem radiographic studies was found within the false lumen on the right side $3 \mathrm{~cm}$ from the origin, establishing the existence of a continuity with the true arterial lumen. The basilar arteries and PICAs were normal.

Microscopic examination showed that the dissections were located between the media and adventitia, and that there was mild thickening of the intima with focal degeneration of the elastica along the entire length of the arteries, consistent with early atheroma. In the left vertebral artery an area of fibrosis was found $1 \mathrm{~cm}$ from its origin, and small vessel formation between the tunica media and adventitia was seen. This was felt to be consistent with a possible old small dissecting aneurysm.

Later examination of entire vertebral arteries in a man of 46 with motor neuron disease, aild a man of 42 with a perforated berry aneurysm of the middle cerebral artery, showed similar thickening of the intima and degeneration of the elastica.

\section{Discussion}

The course of the vertebral artery and its relationship to the neighbouring structures renders it particularly vulnerable to mechanical trauma. The vertebral artery is relatively fixed in its passage through the transverse foramina, and particularly in the region of the atlanto-axial articulation where rotation and tilting of the head and neck largely occurs. ${ }^{48}$ Angiography during head rotation has demonstrated vertebral artery compression at the level of $\mathrm{Cl}$ associated with vertebro-basilar ischaemic symptoms, ${ }^{15}$ and similar occlusion of one vertebral artery has also been shown in asymptomatic volunteers. ${ }^{16}$ The majority of reported vertebral artery injuries which have followed manipulation, yoga, calisthenics, and even spontaneous head turning have occurred in this area. ${ }^{4612}$ Such activities have usually involved cervical hyperextension, rotation, or both.

We confirm that the vertebral arteries are particularly vulnerable at the level of the atlanto-axial articulation, where bilateral dissections between the media and adventitia were found. Importantly, the presence of a communication between the lumen and the origin of the dissecting plane of the right vertebral artery was demonstrated, suggesting that the haematoma arose from within the vessel lumen rather than from the vasa vasorum. The pathological findings also indicate that the vertebral arteries may be vulnerable at a point close to their origins, before they enter the transverse foramina. Such proximal dissections may be missed in radiographic examinations because of their position. Perhaps because of this, and the infrequency of pathological examination of the entire length of the vertebral arteries, extra-cranial vertebral artery dissections have only rarely been described. ${ }^{17}$

Controversy persists as to what degree are cerebral artery dissections spontaneous or traumatic. Some have related all such cases to trauma, often as minimal as occurs with cervical rotation. In others, various conditions predisposing to arterial injuries have been cited: the presence of cervical osteophytes compressing the vertebral artery, ${ }^{6}$ atherosclerosis, ${ }^{6}$ congenital or acquired defects of the media, ${ }^{18}$ syphilis, ${ }^{19}$ fibroelastic intimal abnormalities, ${ }^{20}$ homocystinuria, ${ }^{21}$ and fibromuscular dysplasia. ${ }^{22}$ However, as in our patient where the only finding was early atheroma consistent with the patient's age, most patients have been young adults with normal anatomy and no demonstrable predisposing cause.

Clinical manifestations are influenced by the extent and nature of injury and the available collateral circulation. The onset of symptoms may occur late, often over 24 hours after the injury. ${ }^{12}$ Temporary occlusion or minor injury to one or both vertebral arteries may lead to transient brainstem ischaemia, which is likely to be the most common result of the vascular injury. The occurrence of such episodes is a warning sign that has sometimes been ignored in the history of patients who develop more serious complications when manipulation is repeated. ${ }^{710}$

Lateral medullary infarction has been the most frequent stroke syndrome described, ${ }^{2-14}$ but sudden death, quadriplegia, and the locked-in syndrome have also been reported. ${ }^{269}$ In addition, patients with dissection involving the intradural portion may present with a subarachnoid haemorrhage. ${ }^{23-26}$

Radiographic findings may be diverse, ranging from complete occlusion to irregular arterial narrowing of a variable extent with or without accompanying dilatation. ${ }^{4-681724-26}$ The findings in this case suggest that the full length of the vertebral arteries, including their origins, should be examined.

The optimal treatment for patients with dissecting aneurysm, traumatic or spontaneous, has not been determined. A conservative approach to neurologically stable patients has been recommended. 412172527 In the case of unilateral injury collateral circulation may be adequate, and spontaneous resolution has been shown radiographically. ${ }^{1727}$ Anticoagulant therapy has been advocated in order to prevent thrombosis of the true lumen or distal embolisation from a mural thrombus. ${ }^{461727}$ However, this treatment has not been widely accepted because of the occurrence of vessel rupture with perivascular $^{4}$ or subarachnoid haemorrhage, ${ }^{23-26}$ 
and the possibility of such therapy extending preexisting dissection, or transforming an infarction into an intracerebral haemorrhage. ${ }^{28}$

In those with progressive signs, surgery, including revascularisation procedures and proximal arterial occlusion, has been tried. ${ }^{24-26}$ However, the exact indications for surgery and its advantages over conservative treatment have yet to be established.

We thank Dr M Sadka for permission to report this case and Miss Gail Della Torre for secretarial assistance.

\section{References}

1 Cameron RJ. Australian Health Survey 1977-8: Consultations with Health Professionals. No. 4322.0. Canberra: Australian Bureau of Statistics. 1981.

2 Pratt-Thomas HR, Berger KE. Cerebellar and spinal injuries after chiropractic manipulation. JAMA 1947;133:600-3.

3 Ford FR, Clark D. Thrombosis of the basilar artery with softenings in the cerebellum and brainstem due to manipulation of the neck. Bull Johns Hopkins Hosp 1956;98:37-42.

4 Sherman DG, Hart RG, Easton JD. Abrupt change in head position and cerebral infarction. Stroke 1981;12:2-6.

5 Simmons KC, Soo YS, Walker G, Harvey P. Trauma to the vertebral artery related to neck manipulation. Med J Aust 1982;1:187-8.

6 Schellhas KP, Latchaw RE, Wendling LR, Gold LH. Vertebrobasilar injuries following cervical manipulation. JAMA 1980;244:1450-53.

7 Smith RA, Estridge MN. Neurologic complications of head and neck manipulations. JAMA 1962;182; 5:528-31.

8 Mueller S, Sahs AL: Brainstem dysfunction related to cervical manipulation. Neurology 1976;26:547-50.

9 Horn SW. The "Locked-in" syndrome following chiropractic manipulation of the spine. Ann Emerg Med 1983;12:648-50.

10 Dumas $\mathrm{R}$ et Guard O. Les accidents vasculaires du tronc cerebral survenant a la suite de manipulations cervicales. Ann Med Phys 1979;22;1:62-70.

11 Beatty RA. Dissecting haematoma of the internal carotid artery following chiropractic cervical manipu- lation. J Trauma 1977;17;3:248-9.

12 Dragon R, Saranchak H, Lakin P, Strauch G. Blunt injuries to the carotid and vertebral arteries. $\mathrm{Am} \mathrm{J}$ Surg 1981;141:497-500.

13 Green D, Joynt RJ. Vascular accidents to the brainstem associated with neck manipulation. JAMA 1959;170:522-4.

14 Schmitt HP. Rupturen und thrombosen der arteria vertebralis nach gedeckten mechanischen insulten. Schweiz Arch Neurol Neurochir Psychiatr 1976;119:363-79.

15 Barton JW, Margolis MT. Rotational obstruction of the vertebral artery at the atlanto-axial joint. Neuroradiology 1975;9:117-20.

16 Faris AA, Poser CM, Wilmor DW, Agnew CH. Radiologic visualisation of the neck vessels in healthy men. Neurology 1963;13:386-96.

17 Goldstein SJ. Dissecting hematoma of the cervical vertebral artery. J Neurosurg 1982;56:451-4.

18 Takita K, Shirato H, Akasaka T, Hukazawa H. Dissecting aneurysm of the vertebro-basilar artery. No To Shinkei 1979;31:1211-8.

19 Scholefield BG. A case of aneurysm of the basilar artery. Guys Hosp Rep 1924;74:485-7.

20 Berkovic SF, Spokes RL, Anderson RM, Bladin PF. Basilar artery dissection. $J$ Neurol Neurosurg Psychiatry 1983;46:126-9.

21 Grieco AJ. Homocystinuria: pathogenetic mechanisms. Am J Med Sci 1977;273:120-32.

22 Hegedus KH, Nemeth G. Fibromuscular dysplasia of the basilar artery. Arch Neurol 1984;41:440-2.

23 Farrell MA, Gilbert JJ, Kaufman JC. Fatal intracranial arterial dissection: clinical pathological correlation. $J$ Neurol Neurosurg Psychiatry 1985;48:111-21.

24 Yonas H, Agamanolis D, Takaoka Y, White RJ. Dissecting intracranial aneurysms. Surg Neurol 1977;8:407-15.

25 Berger MS, Wilson CB. Intracranial dissecting aneurysms of the posterior circulation. $J$ Neurosurg 1984;61:882-94.

26 Friedman AH, Drake CG. Subarachnoid haemorrhage from intracranial dissecting aneurysm. J Neurosurg 1984;60:325-34.

27 Shuster E, Mokri B, Sundt TM, Houser OW, Piepgras D. Spontaneous dissection of the internal carotid arteries (Abstract). Neurology 1985;35(Suppl. 1):212.

28 Hochberg FH, Bean C, Fisher CM, Roberson GH. Stroke in a 15-year-old girl secondary to terminal carotid dissection. Neurology 1975;25:725-9. 